\title{
Survey: Physicians Generate an Average \$2.4 Million a Year Per Hospital
}

Dallas, TX, February 25, 2019 - While physicians are key providers of medical care at the nation's hospitals, a new survey confirms they also are major drivers of hospital revenue. According to the survey, physicians generate an average $\$ 2,378,727$ per year in net revenue on behalf of their affiliated hospitals.

Conducted by Merritt Hawkins, the nation's leading physician search firm and a company of AMN Healthcare, the survey asked hospital chief financial officers to quantify how much revenue physicians in 18 specialties generated for their hospitals in the previous 12 months. This includes both net inpatient and outpatient revenue derived from patient hospital admissions, tests, treatments, prescriptions, and procedures performed or ordered by physicians.

Cardiovascular surgeons topped the list of physicians examined in the survey. Full-time cardiovascular surgeons generate an average of nearly $\$ 3.7$ million a year on behalf of their affiliated hospitals, according to the survey, followed by invasive cardiologists at $\$ 3.5$ million, neurosurgeons at $\$ 3.4$ million and orthopedic surgeons at $\$ 3.3$ million.

"The value of physician care is not only related to the quality of patient outcomes," said Travis Singleton, Merritt Hawkins Executive Vice President. "Physicians continue to drive the financial health and viability of hospitals, even in a healthcare system that is evolving towards valuebased payments."

It is not just physician specialists who generate high dollar volumes for hospitals, the survey indicates. Family physicians generate an average of $\$ 2.1$ million in net revenue annually for their affiliated hospitals, while general internists generate an average of almost \$2.7 million.

The average net revenue generated by all physicians included in the survey $(\$ 2,378,727)$ is up $52 \%$ from 2016, the last year Merritt Hawkins conducted the survey. Average revenue generated by each of the 18 medical specialties included in the survey increased compared to 2016, in most cases significantly. 


\section{More Outpatient Visits, Higher Costs, More Acuity}

While the number of hospital inpatient stays has decreased or remained flat in recent years, the cost per hospital stay has increased, said Singleton, one factor that may be driving the comparatively high revenue averages generated by physicians. In addition, the number of hospital outpatient visits has more than tripled since 1975 and the average cost of these visits has grown, a further reason for physician revenue increases, according to Singleton. Patient demographics and patient acuity also are playing a role. As the population ages and as patient acuity increases, utilization of healthcare services provided by or generated by physicians also increases, as do physician generated revenues.

"Demographics are our destiny," said Singleton. "New delivery models that promote prevention, population health and fee-for-value are laudable innovations but they don't change the basic facts. People get older and require more medical care, with much of it ordered by or directly provided by physicians."

\section{Cost/Benefits}

The survey also provides a cost/benefits analysis showing which physicians provide the best return on investment by comparing salaries in various medical specialties to revenue generated by physicians in those specialties. Family physicians, for example, average a starting salary of $\$ 241,000$, according to Merritt Hawkins' data, while generating nine times that much in hospital revenue. Orthopedic surgeons average $\$ 533,000$ in starting salary while generating six times that much in hospital revenue.

"Primary care physicians such as family physicians represent an excellent return on investment, which is one reason they have been our number one search for the last 12 years" Singleton said.

A copy of the complete survey report is available by calling Merritt Hawkins at $800-876-0500$ or by accessing www.merritthawkins.com.

\section{About Merritt Hawkins}

Merritt Hawkins is the largest physician search and consulting firm in the United States and is a company of AMN Healthcare (NYSE: AMN). More information about Merritt Hawkins can be accessed at www.merritthawkins.com.

\footnotetext{
About AMN Healthcare

AMN Healthcare is the leader and innovator in healthcare workforce solutions and staffing services to healthcare facilities across the nation. The Company provides unparalleled access to the most comprehensive network of quality healthcare professionals through its innovative recruitment strategies and breadth of career opportunities. With insights and expertise, AMN Healthcare helps providers optimize their workforce to successfully reduce complexity, increase efficiency and improve patient outcomes. AMN delivers managed services programs, healthcare executive search solutions, vendor management systems, recruitment process outsourcing, predictive modeling, mid-revenue cycle solutions, and other services. AMN Healthcare is committed to fostering and maintaining a diverse team that reflects the communities we serve. Our commitment to the inclusion of many different backgrounds, experiences and perspectives enables our innovation and leadership in the
} 
healthcare services industry. For more information about AMN Healthcare, visit www.amnhealthcare.com.

\#\#\# 